\title{
Hospitalización a Domicilio en hospitales públicos gallegos
} Hospital at home units in galician public hospitals

\author{
ML Hermida Porto, LM Dopico Santamariña, F Lamelo Alfonsín, \\ B Aldámiz-Echevarría Iraurgui, MA Silva César, L Vidán Martínez \\ Unidad de Hospitalización a Domicilio. Complexo Hospitalario Universitario A Coruña. SERGAS. A Coruña
}

\begin{abstract}
Resumen
Objetivo. Describir la organización y la actividad que realizan las Unidades de Hospitalización a Domicilio (HADO) en los hospitales públicos gallegos.

Material y métodos. Hemos realizado una encuesta detallada sobre la actividad, organización, docencia, relación con atención primaria..., basándonos en los datos de la actividad del 2013, a cada una de las unidades del Sistema Público. Se realizó un estudio descriptivo de las variables expresando las medidas de centralización y dispersión de las variables cuantitativas y, en caso de las cualitativas, en valor absoluto y porcentaje.

Resultados. Participaron las 10 unidades de Hospitalización a Domicilio, de la red pública de Galicia, en activo durante el año 2013, lo que representa el $78 \%$ de los centros hospitalarios. El 5.92\% de las camas hospitalarias pertenecen a estas unidades, con una media de 12 camas por facultativo. Hay 38 médicos trabajando en esta modalidad de los que el $50 \%$ son Médicos de Familia y el $31.7 \%$ Internistas. Se realiza un 17.13\% de ingresos directamente desde Atención Primaria. Del total de ingresos anuales, 5514, el 27\% eran pacientes paliativos oncológicos.

Conclusiones. HADO es un modelo de asistencia alternativo en determinados casos a la hospitalización convencional, con una gran heterogeneidad entre cada una de las Unidades pero con una clara vocación en el cuidado en casa de pacientes paliativos oncológicos y no oncológicos, así como en exacerbación de patología crónica y patología infecciosa aguda.

Palabras Clave: Hospitalización a domicilio; Manejo clínico; Médicos de familia; Internistas; Alternativas a la hospitalización; Cuidados paliativos; Enfermedades crónicas.
\end{abstract}

\section{Introducción}

La Hospitalización a Domicilio se caracteriza por proporcionar, en la vivienda del paciente, niveles de diagnóstico, cuidados y tratamientos de intensidad y/o complejidad equiparables a los dispensados en el hospital. Es proporcionada por profesionales de salud especializados, durante un tiempo limitado, a pacientes que de otra manera hubiesen precisado atención en un hospital de agudos ${ }^{1}$, sirviendo de puente entre Atención Primaria y Especializada.

Comparada con la hospitalización tradicional, HADO ha demostrado que obtiene, como mínimo, similares resultados clínicos, pero con una importante mejora en la calidad de vida del enfermo y de sus familiares ${ }^{2}{ }^{3}$.

La primera Unidad de Hospitalización a Domicilio de España comenzó su andadura en el año 1981 en el Hospital provincial de Madrid, creándose la primera gallega en el año 1987 en A Coruña. La transferencia de competencias en materia de sanidad a las Comunidades Autónomas ha posibilitado en

\section{Abstract}

Objective. To describe the organization and the activities performed by the Hospital at Home Units in Galician public hospitals.

Methods. All Hospital at Home Units of the public system responded a detailed survey about their activities, organization, teaching, and relationships with primary care through year 2013. A descriptive study of the variables was performed, reporting the measures of centralization and dispersion of quantitative variables and, the absolute value and percentage of qualitative ones.

Results. Ten active Hospital at Home Units of Galician public health network during 2013 participated, serving 78\% of the Hospitals in the community. Hospital at Home beds represent $5.92 \%$ of all hospital beds with an average of 12 beds assigned to each physician. A total of 38 physicians were working in this organization model, $50 \%$ of whom were Family Physicians and $31.7 \%$ were Internists. $27 \%$ of total admissions (5514) were palliative cancer patients and $17.13 \%$ of all patients were admitted from primary care facilities.

Conclusions. Hospital at Home Units is an alternative model to conventional hospital care, with a large heterogeneity between each unit but with a clear vocation to home care for palliative oncological and nononcological patients, decompensated patients with chronic diseases, and treatment of stable acute infectious diseases.

Keywords: Hospital at Home; Clinical management; Family physicians; Internists; Alternative to hospitalization; Palliative care; Chronic diseases.

varias comunidades el crecimiento de esta actividad, entre ellas País Vasco, Comunidad Valenciana y Galicia.

En el momento actual contamos en Galicia con 14 unidades en funcionamiento, tres de ellas en el ámbito de hospitales privados y once en la red pública, habiéndose inaugurado la Unidad de Hospital de Burela en Enero de 2014. Todas están formadas por médicos, personal de enfermería y en alguna excepción hay un auxiliar de clínica compartida con otras unidades hospitalarias.

Un paciente podría ingresar en HADO si reúne una serie de criterios médicos y sociológicos para asegurar que en todo momento se mantenga un nivel de calidad similar al que se ofrece en el hospital:

- Domicilio del paciente en zona de cobertura de HADO.

- Aceptación del ingreso en HADO por el paciente o por la persona responsable.

- Existencia de un cuidador principal identificado que se responsabilice del paciente. 
- Teléfono de contacto en el domicilio.

- Cumplimiento de criterios clínicos de ingreso en HADO: diagnóstico establecido, estabilidad clínica y posibilidad de realizar tratamiento en domicilio (según la frecuencia de los fármacos y características de cada unidad)

Cuando nos referimos a diagnóstico establecido buscamos intentar no tener que desplazar al paciente al hospital constantemente para realizar pruebas complementarias, aunque siempre que sean precisas se realizarán como en cualquier otro servicio del Hospital. El criterio de estabilidad clínica hace referencia a que, a priori, no ingresaría un paciente que precisa vigilancia continua por inestabilidad hemodinámica, respiratoria, etc.

Una vez que se identifica un posible candidato se inicia la valoración del enfermo. Se examina la historia clínica, su estado clínico y el plan terapéutico inicial para su seguimiento en la Unidad. La entrevista con el enfermo y la familia permite confirmar si HADO es recomendable, al comprobar que se cumplen los requisitos imprescindibles y que, una vez informado, el enfermo acepta la propuesta. La selección es realizada por los profesionales de la Unidad. Es de suma importancia conocer la disponibilidad de la persona que desempeñará la función de cuidador; ya que es a ella a quien se le confía la atención general del enfermo en su domicilio, así como la implicación en diferentes actuaciones como administrar medicación subcutánea, controles glucémicos.... Finalmente, se produce el traslado al domicilio para seguir el proceso asistencial previsto bajo la responsabilidad del equipo de HADO.

En los días sucesivos, el equipo asistencial realizará las actuaciones pertinentes en el domicilio del enfermo. El seguimiento clínico es análogo al del hospital. El enfermo dispone de la comunicación telefónica con la Unidad y, en las horas que esta no está operativa, con el 061. Cuando se estima oportuno, es dado de alta con la correspondiente programación de visitas al médico de Medicina Familiar y al facultativo del hospital ${ }^{4}$.

Siguiendo estas premisas planteamos este artículo con el objetivo de mostrar en qué consisten y la actividad que realizan las Unidades de Hospitalización a Domicilio (HADO) en Ios hospitales públicos gallegos. La finalidad es obtener una visión de la organización y actividad que se realiza en nuestra Comunidad Autónoma, al tiempo que acercar HADO y sus posibilidades a los Servicios de Medicina Interna.

\section{Material y métodos}

Realizamos una encuesta que se envió a los jefes de cada Unidad en abril de 2014, recibiendo las respuestas hasta septiembre. Las unidades encuestadas fueron las de los hospitales de Lugo (Hospital Universitario Lucus Augusti), Ferrol (Hospital Arquitecto Marcide), A Coruña (Complexo Hospitalario Universitario A Coruña), Cee (Hospital Virxe da Xunqueira), Santiago de Compostela (Complexo Hospitalario Universitario de Santiago), Barbanza (Hospital da Barbanza), Pontevedra (Complexo Hospitalario Universitario de Ponteve- dra), Salnés (Hospital do Salnés), Vigo (Complexo Hospitalario Universitario de Vigo) y Ourense (Complexo Hospitalario Universitario de Ourense), no enviándose a la Unidad del Hospital comarcal de Burela porque inició su actividad en Enero de 2014.

El tipo de cuestionario permitía respuestas abiertas, agrupándose en 8 categorías:

1. Organización de la Unidad incluyendo el número total de camas del centro y de HADO, número de pacientes por adjunto, cobertura horaria y geográfica, estancia media, pacientes al año, tasa de reingreso y dependencia jerárquica.

2. Personal preguntándose por número de médicos en plantilla, Jefes de Unidad, número de enfermeras, organización de los equipos, residentes que rotan y obligatoriedad o no de la rotación.

3. Organización de los turnos/cobertura averiguando como está organizado el sistema de turnos, cobertura horaria, existencia de guardias.

4. Interconsultas. En este punto cuantificamos el número de interconsultas anuales y cómo se derivan los pacientes a la unidad, si hay protocolos específicos y quién hace las interconsultas.

5. Relación con la Universidad preguntando sobre rotaciones de alumnos en las Unidades.

6. Relación con Atención Primaria y la posibilidad de derivar pacientes directamente, detallando número, medio de derivación y tipo de patología.

7. Principales campos de acción. Aquí incidimos en qué tipo de pacientes ingresan en HADO, diagnósticos principales, porcentaje de paliativos, método de administración de antibióticos (personal de la unidad, autoadministración, mixto)

8. Consultas externas en la que preguntamos por la existencia de consultas externas asociadas a la unidad.

Se recogieron todos los datos procesándose en hoja de cálculo Excely se realizó un estudio descriptivo de las variables expresando las medidas de centralización y dispersión de variables cuantitativas y el valor absoluto y porcentaje de las cualitativas.

\section{Resultados}

En los hospitales públicos gallegos no se han implantado unidades de HADO en el Hospital comarcal de Valdeorras, Hospital comarcal de Monforte ni en el Hospital de Verín, habiéndose incorporado el Hospital da Costa (Burela) a principios de 2014. Todos los demás hospitales tienen en su cartera de servicios Hospitalización a Domicilio.

El número total de camas que implican los hospitales del estudio son 6196, de las que el 5.92 \% pertenecerían a nuestras Unidades (396 camas), en donde los hospitales de más de 600 camas tienen aproximadamente 50 habilitadas en esta modalidad y el resto, por debajo de 22 (variando desde las 11 del Salnés hasta las 60 de Vigo). Existe mayor homogeneidad 
en el número de camas por adjunto, siendo de unas 12, excepto en Pontevedra que pueden llegar hasta 24.

La mayoría de los médicos que trabajan en las Unidades, 38 en total, pertenecen a especialidades de Medicina Familiar y Comunitaria (50\%) o Medicina Interna (31.57\%), habiendo también generalistas, psiquiatras, cirujanos, UCl o geriatras. En Hospitales como el de Lugo y el de Santiago tienen 7 médicos en plantilla pero, en ambos casos, parte de ellos trabajan en otra actividad: 3 en el Hospital de Día Polivalente y Unidad de Paliativos en el primer caso y 4 en la Unidad de Media Estancia (UME) y en Paliativos en Santiago. Del resto de las unidades hay 2 unipersonales en Ferrol y Salnés, con 2 facultativos en Cee, Barbanza y Pontevedra y de 5 en A Coruña, Ourense y Vigo. En las unidades de 20 menos médicos no está nombrado un jefe de unidad, excepto en Pontevedra. Todas las unidades dependen directamente de la Dirección médica excepto en el Salnés que depende del Servicio de Urgencias y en el Barbanza del Servicio de Medicina Interna.

El otro pilar fundamental en HADO es la enfermería. El número total de enfermeras adscritas en Galicia es de 51, con un ratio médico/enfermera de 1,7 enfermeras por médico, proporción que es mayor en los hospitales con menos médicos (1:2), y que disminuye en los de más personal (0.62 :1) (Figura 1)

Figura 1.- Personal sanitario de las Unidades de HADO gallegas

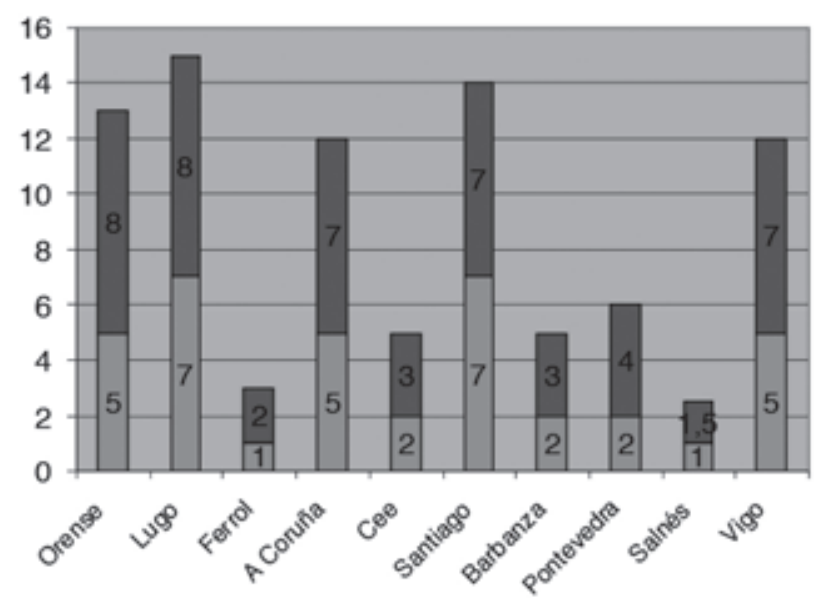

- Personal de enfermeria

$\square$ Médicos

En las unidades se trabaja por las mañanas en equipos formados por un médico y un DUE, habiendo, en muchos casos, al menos un equipo formado únicamente por personal de enfermería para realizar procedimientos y visitas que no precisen al médico ese día, sucediendo lo mismo en el horario en que no hay cobertura médica pero sí de enfermería. En A Coruña el médico y la enfermera realizan la atención de forma individual.

Las principales dificultades de la Hospitalización a domicilio residen en la cobertura geográfica y la cobertura horaria.
La cobertura geográfica no es universal, pudiendo evidenciarse su distribución en el mapa adjunto (Figura 2). Esto está limitado por el número de equipos trabajando en cada zona, los tiempos de desplazamiento y el volumen de la población diana.

Figura 2.- Cobertura geográfica de la Unidades de HADO gallegas

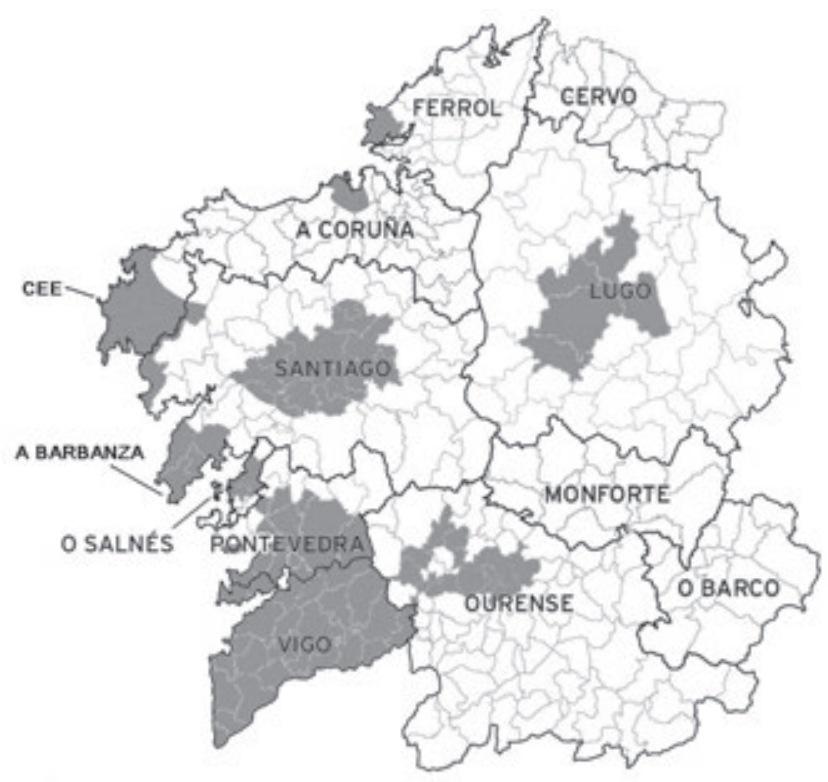

La cobertura horaria (Tabla 1) presenta una gran variabilidad, lo cual depende de las direcciones hospitalarias, dificultando el poder estandarizar ciertas actividades, admitir cierto tipo de pacientes... Lo que sí está unificada es la cobertura fuera de nuestro horario, que es realizada por el 061.

Tabla 1. Horario de cobertura médico y de enfermería en las distintas HADOS

\begin{tabular}{|c|c|c|}
\hline Hospital & Médico & Enfermería \\
\hline Barbanza & Lunes a Viernes de 8-15 h & $\begin{array}{l}\text { Lunes a Viernes de 8-21 h } \\
\text { Sábados de 8-14 h }\end{array}$ \\
\hline Cee & Lunes a Viernes de 8-15 h & $\begin{array}{l}\text { Lunes a Viernes de 8-21 h } \\
\text { Sábados de 8-15 h }\end{array}$ \\
\hline A Coruña & $\begin{array}{l}\text { Lunes a Viernes de 8-21 h } \\
\text { Sábado y Domingo de 8-15 h }\end{array}$ & $\begin{array}{l}\text { Lunes a Viernes de 8-21 h } \\
\text { Sábados y domingos de 8-15 h }\end{array}$ \\
\hline Orense & Lunes a Domingo de 8-21 h. & Lunes a Domingo de 8-21 h \\
\hline Salnés & Lunes a Viernes de 8-15h & $\begin{array}{l}\text { Lunes a viernes de } 8-21 \text { h } \\
\text { Sábados de } 8 \text { a } 15 \text { h }\end{array}$ \\
\hline Ferrol & Lunes a Viernes de 8-15 h & Lunes a Viernes de 8-15 h \\
\hline Pontevedra & Lunes a Viernes de 8-15 h & Lunes a Viernes de 8-15 h \\
\hline Vigo & Lunes a viernes de $9-21 \mathrm{~h}$ & Lunes a viernes de 9-21 h \\
\hline Lugo & Lunes a sábado de 8-15 h & $\begin{array}{l}\text { Lunes a viernes de 8-21 h } \\
\text { Sábados y domingos de 8-15 h }\end{array}$ \\
\hline Santiago & Lunes a sábado de 8-15 h & Lunes a sábado de 8-15 h \\
\hline
\end{tabular}

Las Unidades de A Coruña y Ourense son las únicas con cobertura médica y de enfermería todos los días del año, aunque no las 24 horas, realizando guardias localizadas el personal facultativo los domingos y festivos. En Unidades como la del Barbanza se tiene pactado que en caso de ha- 
ber fin de semana y festivo el viernes o el lunes trabaja el médico y enfermería ese día.

La estancia media de las Unidades de HADO en el 2013 ha sido de $14.35 \pm 5.96$ días (excluido Santiago) habiendo sido atendidos entre todas las unidades un total de 5514 pacientes (un 2.3\% de todos los ingresos de la comunidad). Con una tasa media de reingresos a los 30 días del $6.17 \%$ (desde el 2,48\% al 16.4\%) En la figura 3 se representan los ingresos por unidad y en la figura 4 las estancias medias.

Figura 3.- Número absoluto de pacientes ingresados en cada HADO en 2013

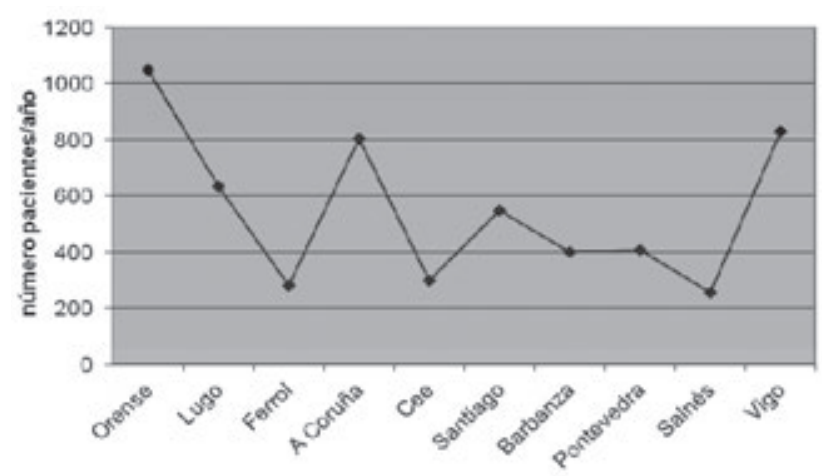

Figura 4.- Estancia media en dícada HADO en 2013

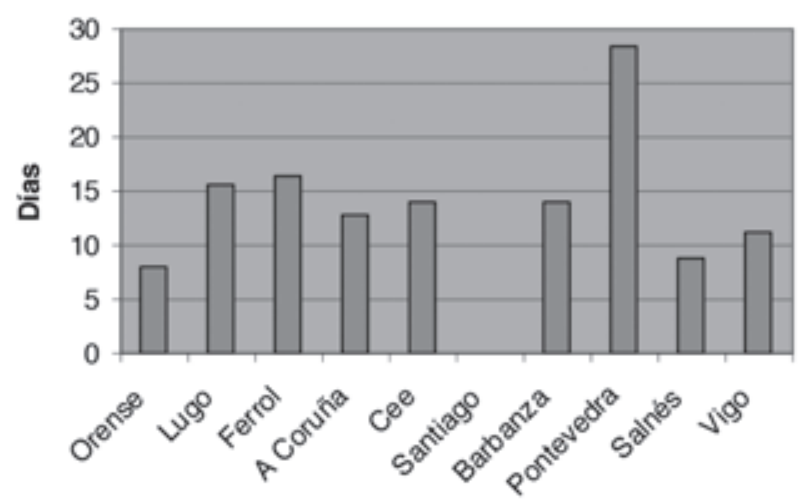

¿De dónde proceden nuestros pacientes y cómo se pueden derivar a nuestras unidades?

En el año 2013, entre todas las Unidades de HADO se realizaron aproximadamente unas 7399 interconsultas, generando, como ya se comentó, 5514 ingresos, esto implica un rechazo del $24 \%$ de las mismas por no cumplir los criterios clínicos o sociológicos antes descritos.

Las interconsultas hospitalarias suele realizarlas el médico al que, según el domicilio del paciente, le corresponda el mismo, puesto que se suele tener subdividida el área de acción en diversas zonas dependientes cada una de un adjunto. Pero hay excepciones como en Santiago en la que las interconsultas las realiza el personal de la UME, en Lugo el Coordinador de la Unidad, en Ourense el Coordinador de la Unidad y la Supervisora, o en A Coruña que desde finales de octubre de 2014 se ha puesto en marcha una unidad de captación y valoración. Respecto a las interconsultas extrahospitalarias, las suelen realizar los propios adjuntos.
Las interconsultas pueden ser divididas en dos categorías: por un lado Atención Primaria y Centros Sociosanitarios y, por otro, Atención Especializada (Figura 5).

Figura 5.- Interconsultas recibidas en cada HAD0 en 2013

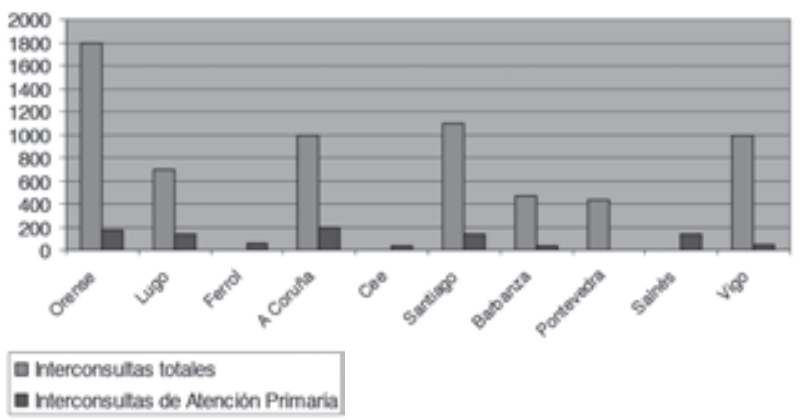

Todas las Unidades permiten el acceso directo de pacientes desde Atención Primaria, siendo el $17,13 \%$ de la actividad de las mismas (Figura 6) La forma de contacto es, en la mayoría de los casos, vía telefónica, habiendo ocasiones que se realizan interconsultas tanto por correo electrónico como por fax. En unidades como la de Lugo se ha desarrollado un protocolo específico para ello. En la Unidad del Barbanza disponen de un protocolo de consulta electrónica. Por otro lado, en varias unidades se han realizado protocolos de Cuidados Paliativos entre HADO, Atención Primaria y Especializada.

Figura 6.- Procedencia de los pacientes ingresados en HADO en 2013

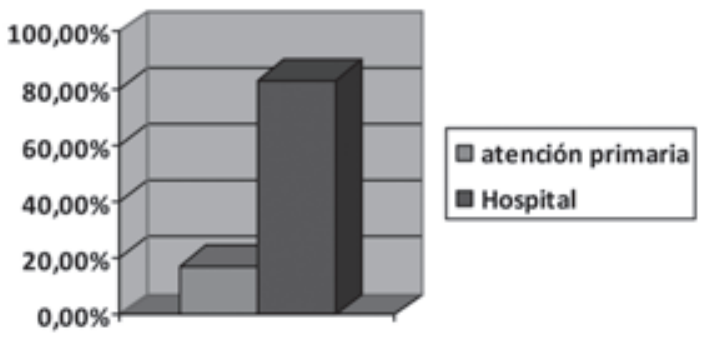

La derivación directa desde Atención Primaria favorece la permanencia del paciente en su domicilio, evitando su derivación a urgencias y posterior ingreso en el régimen de hospitalización convencional, consiguiéndose mayor confort para el paciente y su familia, a la vez que se contribuye a la descongestión del hospital. Los principales diagnósticos de los pacientes derivados en esta modalidad se pueden ver en la tabla 2.

Tabla 2. Principales causas de derivación desde Atención Primaria

\begin{tabular}{l} 
Cuidados paliativos oncológicos \\
Anciano frágil y pluripatológico \\
Infecciones respiratorias \\
Infecciones de úlceras por presión y vasculares \\
Infecciones urinarias \\
Curas complejas \\
\hline
\end{tabular}


Figura 7.- Pacientes paliativos oncológicos respecto al total de pacientes

Por otro lado, el $82.87 \%$ de nuestros pacientes proceden del ámbito hospitalario, tanto de Urgencias, hospitalización convencional, Consultas Externas como Cirugía sin ingreso.

En Unidades como las de Ourense, Santiago, Pontevedra y Vigo se han desarrollado protocolos específicos con Urgencias para la derivación de pacientes. En otras, los días laborables, se acude directamente a Urgencias para realizar los ingresos pendientes y valorar posibles candidatos, como es el caso de A Coruña realizándolo la unidad de captación y valoración, en Ourense el coordinador 0 en el Salnés y Pontevedra los adjuntos de la Unidad. El resto de las Unidades trabajan con Urgencias a través de interconsulta tanto telefónica como en otra modalidad. La tendencia actual es ir incrementando los ingresos desde Urgencias evitando el ingreso convencional, sobre todo en descompensación de crónicos, infecciones respiratorias, infecciones de partes blandas, pielonefritis e infecciones urinarias complicadas en las que se pueden realizar tratamientos endovenosos domiciliarios y seguimiento activo del paciente por parte de la Unidad.

Con el resto de los Servicios se trabaja por medio de interconsultas y valoraciones en planta, habiéndose desarrollado diversos protocolos en cada hospital. (Tabla 3)

Tabla 3. Protocolos implantados en las distintas HADOs gallegas

\begin{tabular}{ll}
\hline Derivación desde Urgencias & Ourense, Santiago, Pontevedra y Vigo \\
\hline Cirugía Mayor Ambulatoria & Lugo \\
\hline Derivación desde Urologia & A Coruña \\
\hline Derivación desde Cirugía Cardiaca & A Coruña \\
\hline Derivación desde UPI & A Coruña \\
\hline Protocolo de Endocarditis & A Coruña \\
\hline Protocolo de TVP & A Coruña \\
\hline Protocolo de Oncología/Paliativos & Santiago de Compostela, Pontevedra \\
\hline Derivación desde Digestivo & Santiago de Compostela \\
\hline $\begin{array}{l}\text { Derivación desde Medicina } \\
\text { Interna }\end{array}$ & Barbanza y Pontevedra \\
Protocolo con Cirugía Vascular & Pontevedra \\
Protocolo con Cirugía General & Pontevedra \\
\hline
\end{tabular}

Los diagnóstico más prevalentes (Tabla 4) son los relacionados con Cuidados Paliativos en paciente oncológicos, 27\% de la actividad (Figura 7), con excepción del Salnés donde la Unidad de Soporte de Cuidados Paliativos hace esa función. Los Paliativos no oncológicos ascienden en unidades como la de Santiago hasta el 33\% de su actividad anual. La otra gran carga asistencial incluye el tratamiento antimicrobiano domiciliario endovenoso $(\mathrm{TADE})^{5}$, por vía periférica o por vía central de acceso periférico o port a cath (Tabla 5).

Con respeto al TADE las modalidades de administración de la medicación pueden ser de tres tipos: 1. administrado siempre por personal de la Unidad, 2. reconstituido por el personal de la unidad y administrado por el paciente 0 cuidador principal tras adiestramiento pertinente, 3. reconstituido y administrado por el paciente o cuidador principal tras

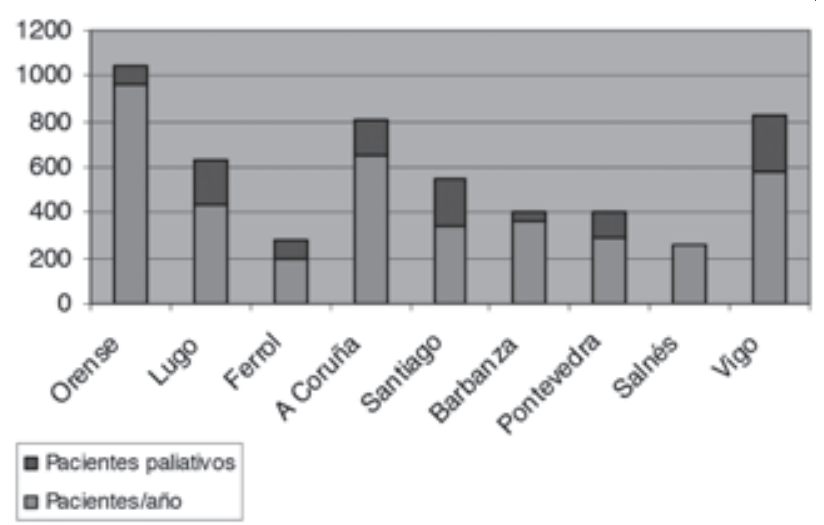

Tabla 4. Grupos diagnósticos más prevalentes

Paliativos oncológicos
Paliativos no oncológicos
Tratamiento antimicrobiano domiciliario endovenoso (TADE)
Soporte hemoterápico
Postquirúrgicos
Crónicos agudizados
Procedimientos: recambios de sondas de gastrostomía, paracentesis
evacuadoras, toracocentesis...)
Nutrición parenteral y enteral
Esclerosis múltiple
Esclerosis lateral amiotrófica
Ventilación mecánica
Programa de crónicos

Tabla 5. Programa TADE: causas de terapia antibiótica domiciliaria endovenosa

Infecciones respiratorias altas y bajas

Fibrosis quísticas sobreinfectadas

Infecciones urinarias: infecciones urinarias complicadas, pielonefritis, abscesos renales, prostatitis, epididimitis, orquitis. .

Infecciones digestivas: diverticulitis, abscesos hepáticos, colecistitis, abscesos abdominales...

Infecciones osteoarticulares: bursitis, sinovitis, artritis sépticas, osteomielitis, infecciones de material protésico ..

Endocarditis infecciosas

Neutropenias

VIH e infecciones oportunistas

Infecciones de partes blandas: celulitis, linfangitis, úlceras

sobreinfectadas

el adiestramiento pertinente. En cualquiera de los tres casos siempre se garantiza acceso a nuestro personal y vigilancia de las vías y complicaciones. En nuestra comunidad hay unidades como las de Ourense, Pontevedra, Vigo o Ferrol en las que la administración siempre lo realiza el personal de la Unidad salvo en excepciones muy puntuales. En el Barbanza y Salnés, por norma, se enseña la autoadministración. En las demás unidades se utilizan sistemas mixtos dependiendo del tipo de paciente/familia, y de la frecuencia de administración del antibiótico, usándose, en ocasiones, infusores. 
Finalmente, la mayoría de las Unidades (excepto Cee) participan en la formación MIR con la rotación de 80 residentes, sobre todo, de Medicina Familiar y Comunitaria y, en menor medida, de Medicina Interna, Geriatría y Radioterapia. La rotación es obligatoria para los médicos de familia que se forman en los hospitales de A Coruña, Lugo, Ferrol y Santiago de Compostela. La duración de la rotación es variable según la unidad docente y la especialidad, siendo de un mínimo de una semana hasta 2 meses. También en los hospitales de Vigo, Santiago y A Coruña rotan alumnos de la Facultad de Medicina.

\section{Discusión}

Estamos en una de las cuatro Comunidades Autónomas en las que en el mayor número de hospitales de la red pública (78\%) se ha implantado este modelo de atención al paciente, la Hospitalización a Domicilio, por detrás de La Rioja, Comunidad Valenciana y País Vasco.

Esto no significa que se consiga dar dicha asistencia a toda la población, siendo las áreas perihospitalarias las privilegiadas y las zonas más distantes, con mayor dificultad y tiempos de desplazamientos a los mismos, las no cubiertas, por lo que no se cumple el principio de equidad en el acceso a dicha actividad.

Como se ha podido observar cada unidad tiene su propia organización en cuanto a número de personal facultativo y DUE, horario, forma de trabajar y cartera de servicios, dependiendo todo ello del hospital al que pertenezca, las circunstancias y necesidades locales existentes.

Por tradición, la mayoría del personal sanitario y no sanitario asocian la Hospitalización a Domicilio con los Cuidados Paliativos. En el Plan Galego de Coidados Paliativos ${ }^{6}$ aparecemos, junto a los Equipos de Soporte de Cuidados Paliativos, como el tercer pilar del plan asistencial, siendo las otras dos Atención Primaria y las Unidades de Cuidados Paliativos. Como se evidencia, por los datos aportados, es una parte importante de nuestra actividad diaria y desde la filosofía que plantea la Consellería en el documento estrategias 20146 de garantizar la prestación de servicios en el lugar 0 en el momento adecuados. Consideramos esta actividad como una de nuestras prioridades, atendiendo a pacientes con dificultades en el control sintomático y alivio del sufrimiento físico, emocional y/o psicosocial, acompañando en el proceso al paciente y la familia ${ }^{7}$.

Como se puede observar, la actividad de HADO abarca muchos más conceptos y patologías sobre las que hemos hecho referencia. Cada vez tiene más impronta en nuestra actividad diaria el paciente agudo estable que precisa medicación endovenosa como diuréticos, corticoides 0 antibioterapia, nebulizaciones... Este tipo de paciente se beneficia de estar en su domicilio con una asistencia clínica proporcionada por personal hospitalario. Estas medidas permiten las tres líneas de trabajo que se están desarrollando en muchos de nuestros hospitales:

1. Evitar ingresos convencionales ingresando directamente desde Urgencias a HADO tras estabilizar al paciente;

\section{Altas precoces de planta,}

3. Valoraciones en el domicilio directamente tras derivación tanto de Atención primaria como de centros Socio-sanitarios como desde Consultas Externas u Hospitales de Día.

Estas tres vías permiten liberar y aumentar la rotación de camas físicas en un sistema de por sí fácilmente colapsable, disminuir la incidencia de complicaciones (infecciones nosocomiales multirresistentes, deliriums, desorientaciones...), reduciendo el estrés familiar y del paciente, con un nivel de satisfacción muy elevado ${ }^{8}$. Todo ello con una baja tasa de reingresos en el hospital durante el siguiente mes por la misma patología, y una mediana de estancia de 14 días (teniendo en cuenta el ingreso de paliativos, antibioterapia de larga evolución, curas complejas...).

Con todo ello el nivel de complejidad de cuidados ha ido incrementándose paulatinamente desde la apertura en 1989 de la primera unidad en A Coruña.

En el momento actual consideramos la necesidad de tender puentes hacia todos los niveles asistenciales y seguir siendo nexo entre el medio hospitalario y Atención Primaria, siendo en este último nivel asistencial espacio importante donde incidir e implementar protocolos de derivación para patologías infecciosas, paliativos...

La principal limitación de este estudio deriva de la propia naturaleza del método de obtención de los datos, y el hecho de que la mayoría de nuestras gerencias no tienen codificados muchos de nuestros datos. A pesar de ello, su valor estriba en ser el primer trabajo realizado en Galicia que analiza y da a conocer nuestras unidades así como los diferentes aspectos organizativos y cartera de servicios.

Los resultados nos indican que nuestra incidencia en la comunidad es pequeña tanto en número de pacientes atendidos como área geográfica comprendida, pero el trabajo realizado por los 38 facultativos y 51 DUE adscritos a HADO en Galicia, ayudan a dar una mejor calidad asistencial en nuestro sistema sanitario.

\section{Bibliografía}

1. Sanroma P, Sampedro I, González CR, Baños MT. Recomendaciones clínicas y procedimientos.Hospitalización Domiciliaria. Santander: Fundación Marqués de Valdecilla; 2011.

2. Regalado de Los Cobos J, Aizpuru Barandiaran F, Oceja Barrutieta E, de Juan Rodríguez M, Apraiz Ruiz L, Altuna Basurto E, Gomez Rodríguez de Mendarozqueta M, LópezPicado A, Cia Ruiz JM. Tratamiento de la neumonía adquirida en la comunidad en hospitalización a domicilio: resultado clínico en casos con diferente nivel de gravedad. Med Clin (Barc). 2010 Jul 12;135(2):47-51

3. Mirón Rubio M. Modelo de hospital a domicilio como una alternativa eficaz en el manejo de la insuficiencia cardiaca crónica descompensada. Rev Clin Esp. 2010 May $5 ; 210: 244$

4. Cuxart Melich A, Estrada Cuxart 0. Hospitalización a domicilio: oportunidad para el cambios. Med Clin (Barc). 2012;138(8):355-360.

5. Mirón Rubio M, Estrada Cuxart 0,González Ramallo VJ. Protocolos: Tratamiento antimicrobiano domiciliario endovenos. Sociedad Española de Medicina Interna. 2008.

6. Estrategia Sergas 2014, la sanidad pública al servicio del paciente. Xunta de Galicia. Consellería de Sanidades 2012.

7. Plan Galego de coidados paliativos. Xunta de Galicia. Consellería de Sanidade 2006.

8. Llorente Díez MB, Salmón García MB, Escudero Berasategui JM. Encuesta de satisfacción en hospitalización convencional vs hospitalización a domicilio. Complejo Hospitalario de Navarra. Congreso Nacional de Hospitalización a Domicilio, Burgos, Junio 2014. 